\title{
To the problem of ensuring stability of activities of construction companies engaged in high-rise building construction
}

\author{
Nikolay Ivanov ${ }^{1, *}$, and Ahmed Safe Aldeen ${ }^{1}$ \\ ${ }^{1}$ Moscow State University of Civil Engineering; 26, Yaroslavskoe Shosse, Moscow, 129337, Russia
}

\begin{abstract}
Recently, more and more attention in scientific literature has been drawn to improving the sustainability of organization. The growth in the volume of high-rise construction in Russia makes the task of assessing and ensuring the sustainability of organizations and enterprises leading this type of construction very relevant. The article considers the approach to assessing the sustainability of the organization's activities in the context of functioning of quality management system (QMS). It puts forward the hypothesis that assessment of sustainability of an organization that has a real and efficient functioning quality management system can be based on the results of assessing the effectiveness of the QMS. The article describes in sufficient detail the sequence of actions to form a list of criteria for assessing the effectiveness of the QMS and sustainability of the organization, and to evaluate both characteristics on the basis of these criteria. For a clear interpretation of the results obtained, the authors use so-called petal diagrams. It suggests an original approach to their creation and analysis. Based on the results of the study, the authors conclude that in order to assess the sustainability of enterprises and organizations analysis of the dynamics of changes in the basic sustainability factors is mandatory.
\end{abstract}

\section{Introduction}

The current period of development of construction in Russian Federation is characterized by the creation of unique structures and architectural and building complexes, forming increasingly complex construction and operation systems. High-rise construction, original planning and structural solutions of structures based on the achievements of high technologies and modern engineering solutions, represent the progressive tendencies of the modern construction complex.

In these context enterprises and organizations of the sector pay great attention to the quality of housing construction $[1,2]$, to the improvement of the efficiency of management decisions and sustainable development $[3,4,5]$, to the mitigation of risks in construction [6-8]. These issues are especially relevant for design and construction organizations of high-rise construction that have implemented the quality management system (QMS). As noted in [8], the existence of an existing system of quality management and a certificate for

\footnotetext{
*Corresponding author: IvanovNA@mgsu.ru
} 
it indicates that the company can be trusted, that the risks in the company are predictable, and the identified nonconformities are eliminated along with the causes for their occurrence. The basis of any effectively functioning QMS according to the ISO 9001 standard is the principle of continuous improvement. The organization in which the QMS is functioning effectively is in a state of continuous improvement of its activities, and such an organization will always be sustainable. That is why to assess the sustainability of the organization that has implemented the QMS, it is necessary and sufficient to assess the effectiveness of the QMS of this organization. Assessment is proposed to do in the light of the approach outlined below.

\section{Methods}

At the first stage, it is necessary to form a list of indicators on the basis of which an assessment of the effectiveness of the QMS of the organization will be conducted. The actions of this stage are described in detail in a series of publications $[9,10]$. The choice of the initial set of indicators is proposed to be carried out on the basis of the cause-effect "Fishbone" (Ishikawa) diagram [11]. The set of indicators is formed on the basis of the 5M rule that is taking into account the five main factors: machines, materials, man, methods and measurements [12]. For each factor, a set of risks is defined, the manifestation of which is possible during the functioning of the organization, as well as a set of criteria that allows assessing the effectiveness of measures to reduce the impact of these risks on the functioning of the QMS and, as a consequence, on the sustainability of a organization.

The base of the second stage is the application of an expert approach for ranking a set of indicators for assessing the sustainability of the organization and selecting the most significant indicators. A group consisting of $\mathrm{K}$ experts, using the group assessment method (for example, Delphi method or the "Brainstorm" method), forms the final set of criteria for assessing the effectiveness of the QMS functioning for each factor. This set can contain $\mathrm{N}$ indicators, each of which, according to experts' general opinion, reflects in some way the effectiveness of the QMS. Then each expert is invited to order the indicators included in one of the five factors in terms of importance, ranking the indicators of each factor in the range from 1 to $\mathrm{N}$. In this case, the value 1 will correspond to the most significant indicator, and the value of $\mathrm{N}$ will be the least significant. Work with each expert at this step is carried out separately, which allows experts to express their opinions regardless of the opinions of colleagues. As a result of this step, a matrix of expert assessments of the criteria is formed, the rows of which correspond to the criteria being evaluated, and the columns to the experts making the assessment. The matrix element Oij (Table 1) is the numerical value assigned to the $i$-th indicator by the $j$-th expert.

Table 1. Matrix of expert estimates of criteria.

\begin{tabular}{|c|c|c|c|c|c|c|}
\hline & \multicolumn{5}{|c|}{ Experts } \\
\hline Criteria & 1 & 2 & $\ldots$ & $\mathrm{j}$ & $\ldots$ & $\mathrm{K}$ \\
\hline $\mathrm{1}$ & & & & & & \\
\hline 2 & & & & & & \\
\hline $\mathrm{i}$ & & & & & & \\
\hline$\ldots$ & & & & $\mathrm{Oij}$ & & \\
\hline $\mathrm{N}$ & & & & & & \\
\hline & & & & & & \\
\hline
\end{tabular}

Columns are added to the matrix, which reflect the total score of each of the indicators, as well as the average value of each indicator, taking into account the views of all experts. When ranking indicators, experts should take into account both the sectoral features of 
construction and the characteristics of enterprises of high-rise design and construction. An example of a matrix of expert assessments is presented in Table 2.

Table 2. Ranking of indicators within an individual factor.

\begin{tabular}{|c|c|c|c|c|c|c|c|c|c|c|c|c|}
\hline & E1 & E2 & E3 & E4 & E5 & E6 & E7 & E8 & E9 & E10 & Sum & Ave \\
\hline P11 & 3 & 2 & 2 & 3 & 3 & 2 & 3 & 3 & 3 & 2 & 26 & 2,6 \\
\hline P12 & 7 & 8 & 7 & 8 & 8 & 8 & 7 & 6 & 8 & 8 & 75 & 7,5 \\
\hline P13 & 4 & 4 & 5 & 4 & 4 & 4 & 4 & 4 & 5 & 4 & 42 & 4,2 \\
\hline P14 & 8 & 7 & 8 & 7 & 7 & 7 & 8 & 8 & 7 & 7 & 74 & 7,4 \\
\hline P15 & 5 & 6 & 4 & 6 & 5 & 6 & 5 & 5 & 4 & 5 & 51 & 5,1 \\
\hline P16 & 1 & 3 & 1 & 1 & 2 & 3 & 1 & 2 & 2 & 3 & 19 & 1,9 \\
\hline P17 & 6 & 5 & 6 & 5 & 6 & 5 & 6 & 7 & 6 & 6 & 58 & 5,8 \\
\hline P18 & 2 & 1 & 3 & 2 & 1 & 1 & 2 & 1 & 1 & 1 & 15 & 1,5 \\
\hline
\end{tabular}

To judge the degree of consistency of the opinions of experts participating in the ranking of indicators, it is necessary to calculate the coefficient of variation of the estimates of $\mathrm{Vi}$ and the concordance coefficient $\mathrm{W}$. The variation coefficient $\mathrm{Vi}$ is calculated for each i-th index and reflects the degree of agreement of experts' opinions about the relative importance of this indicator:

$$
\mathrm{V}_{\mathrm{i}}=\frac{\sigma_{\mathrm{i}}}{\mathrm{C}_{\mathrm{i}}}
$$

where $\sigma_{\mathrm{i}}=\sqrt{\mathrm{D}_{\mathrm{i}}}$ is the standard deviation of the estimates of each of the indicator and $\mathrm{D}_{\mathrm{i}}=\frac{1}{\mathrm{k}} \sum_{\mathrm{j}=1}^{\mathrm{k}}\left(\mathrm{C}_{\mathrm{ij}}-\mathrm{C}_{\mathrm{i}}\right)^{2}$ is the variance of the estimates.

Estimating expression (1), we can conclude that the smaller the value of $\mathrm{Vi}$, the level of consistency of experts' opinions is higher.

To determine the degree of consistency of the group opinion of experts as a whole for the aggregate of indicators within a certain factor, the concordance coefficient is used:

$$
\mathrm{W}_{\mathrm{i}}=\frac{12 \Delta \mathrm{S}^{2}}{\mathrm{k}^{2}\left(\mathrm{n}^{3}-\mathrm{n}\right)}
$$

where $\Delta \mathrm{S} 2$ - a measure of the degree of consistency of judgments of experts, which is determined by the formula:

Substituting $\Delta \mathrm{S} 2$ in (2), we obtain:

$$
\Delta \mathrm{S}^{2}=\mathrm{k}^{2} \sum_{\mathrm{i}=1}^{\mathrm{n}}\left[\mathrm{r}_{\mathrm{i}}-\frac{\mathrm{n}+1}{2}\right]^{2}
$$

$$
\mathrm{W}_{\mathrm{i}}=\frac{12 \sum_{\mathrm{i}=1}^{\mathrm{n}}\left[\mathrm{r}_{\mathrm{i}}-\frac{\mathrm{n}+1}{2}\right]^{2}}{\mathrm{n}^{3}-\mathrm{n}}
$$

The concordance coefficient can take values from 0 to 1 . For $\mathrm{W}=0$, the experts' opinions practically do not coincide. The value $\mathrm{W}=1$ shows that the experts practically rank the indicators in terms of their importance. The dynamic variation of $\mathrm{W}$ from 0 to 1 reflects an increase in the degree of consensus among experts.

Then the experts again jointly discuss the results and determine the "feature line". By "feature line" we mean the sum of all estimates of one indicator, which is still considered by experts to be worthy of consideration. Indicators with a total score greater than the "feature line" are excluded from further processing. It is proposed to leave six of each factor as significant indicators, thus, the feature line will be the total score of 60 points.

The next step is to process the resulting sample of significant criteria. For each criterion, average expert judgment is determined, and then the rank of the criterion in points is calculated. It is assumed that the sum of the quantities inversely proportional to the average estimates of each criterion corresponds to 100 points, and the rank of each criterion in points is proportional to the value inversely proportional to the average criterion estimate: 
where $i j=\frac{K}{\sum_{j}^{K} \text { Oij }}$.

$$
\mathrm{Ri}=\frac{\mathrm{Bij}}{100} * \sum_{\mathrm{j}=1}^{\mathrm{K}} \mathrm{Bij},
$$

The rank of each criterion is rounded to an integer, subject to the restriction that the sum of the ranks of all criteria is 100 . The total score of all the criteria identified by experts, equal to 100 , corresponds to the "ideal" state of an effectively functioning quality management system. This condition assumes the fulfilment of all the requirements of ISO 9001: 2015.

Finally, the experts again jointly discuss the rules for determining the actual indicators for meeting the requirements of the standard for the selected QMS functioning criteria. For example, for the criterion "Number of claims received from consumers", these rules are presented in Table 3.

Table 3. Rules for adjusting the value of the rank of the criterion.

\begin{tabular}{|c|c|c|}
\hline Criteria name Value range & The range of values & Points \\
\hline \multirow{3}{*}{ Number of claims from } & No claims & Maximum points \\
consumers & Less than 3 claims & Minus 3 points \\
& 3 to 5 claims & Minus 6 points \\
& More than 5 claims & Minus 8 points \\
\hline
\end{tabular}

In the third stage, the sum of the scores of the significant criteria calculated for the current year is compared with the lower limit of the score for the quality management system, the functioning of which can be considered effective. As a rule, according to existing methods of assessing the effectiveness of QMS functioning, the boundary is usually set in the 72-74 points area. If the calculated sum of points of the current year is less than the specified limit, the functioning of the QMS of the construction industry organization is considered ineffective and it is recommended to develop a set of corrective actions aimed at eliminating the causes that reduce the effectiveness of the QMS. Absolute stability of the organization is achieved if all factors have a value of at least 74 points.

\section{Results}

If at least one factor goes beyond the stability line, then it is considered that the organization loses its stability. To assess the general state of the organization, it is necessary to analyze the state of the factor level with the help of a petal diagram, the general form of which is shown in Fig. 1.

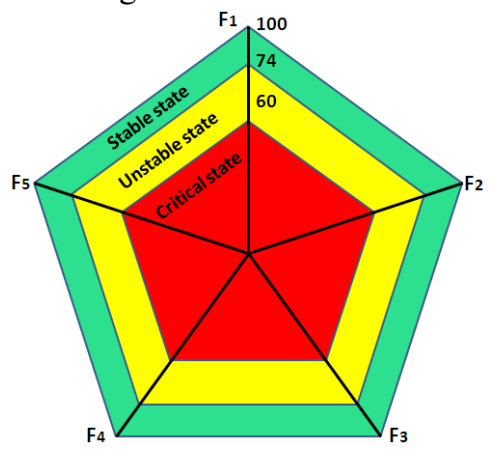

Fig.1. A petal diagram to assess the stability of the organization based on the 5-factor model. 
To interpret the results of assessing the values of factors reflecting the sustainability of the activity of a organization in the construction industry, we introduce three regions of stability: a stable state (green zone), an unstable state (yellow zone), and a critical state (red zone). Define the boundary values for each of these zones. So in the green zone there are factors whose values can range from 100 to 74 points. In the yellow zone, the values of factors are determined from 74 to 60 . The red zone reflects the values of factors from 60 to 0 . Depending on the values adopted, the factors can be unevenly distributed within these three zones. Let's consider the basic variants of an arrangement of values of factors which can arise in the course of an estimation of stability of activity of the organization of highrise construction. Suppose that all factors considered in the current period have values that fall within the range of values of the green zone. In this case, you can call the state of the organization sustainable. This state is the most preferable for any organization, since it speaks of an effectively organized activity. However, in this case the position of the factors within the zone itself is important: factors that are located close to the lower boundary of the zone require special attention from the management of the organization. Otherwise, there is a high probability of an organization moving from a stable state to an unstable state or even a critical state. It is these factors that require targeted impacts through activities aimed at reducing the likelihood of risk occurrence or its complete elimination.

In the conditions of high competition and scarcity in resources that have developed recently in the construction market, the absolutely stable state of the organization for organizations in the construction industry is not a frequent phenomenon. The state where one or more factors are outside the green area in the yellow zone is called unstable. The main task in such a situation is to identify these factors and develop corrective actions for overcoming the unstable state and transition from an unstable situation to a sustainable situation. The selection of optimal measures is important, as a result of which, the organization will not be in an even worse position.

There is a situation in which one or more factors are in the critical red zone. Other factors may be in the yellow or even green zone. In this case, the organization is in a critical, near-bankruptcy state. Such organizations require a special strategy to overcome the crisis situation.

Assessing the stability of an organization at any point in time does not provide exhaustive information about the development of the organization [13]. That is why it is advisable to assess the state of the factors of sustainability in the dynamics: if the value of the factor has a positive dynamic, we can speak about the effectiveness of measures to increase sustainability within this factor. If the value of the factor acquires a border stable state, then one should also evaluate the dynamics, specifying whether it is positive or negative. Particular attention should be paid to those factors, the dynamics of the values of which have a negative value, which corresponds to the tendency to deterioration of stability.

The stability of the organization is proposed to be evaluated by comparing the values of the factors for the current year and the previous year. An example of a diagram reflecting the dynamics of factor values for 2 years (previous and current) is shown in Fig 2. Analysis of the diagram shows that the values of factors F1, F4 and F5 tend to increase, the value of factor F2 decreases, the value of factor F3 remained unchanged. At the same time, despite the increase in the value of factor F4, the stability of the organization within this factor is considered not achieved, in contrast to the values of the factors F1 and F5, within which the organization has taken a stable position. From the results of the analysis it follows that special attention is paid to factors F2 and F4.

On the basis of the obtained data on the dynamics of the efficiency factors of the QMS of a organization, in addition to evaluating each factor separately, it is possible, if necessary, to calculate the integral indicator of the organization's stability. 


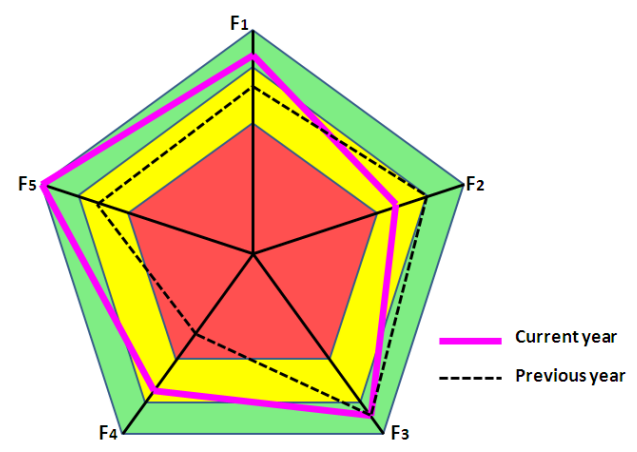

Fig. 2. Dynamics of values of factors reflecting the stability of the organization.

\section{Discussion}

As noted in [14], in the general case, the complex stability of the organization of the construction industry can be described by the following function:

$$
y(t)=f\left\{F_{1}(t) ; F_{2}(t) ; F_{3}(t) ; F_{4}(t) ; F_{5}(t)\right\}
$$

where $\mathrm{F}_{1}(\mathrm{t}), \mathrm{F}_{2}(\mathrm{t}), \ldots, \mathrm{F}(\mathrm{t})$ are the factors influencing the complex stability of the organization.

The interpretation of the values of the complex stability function is presented in Table 4 .

Table 4. Interpretation of the values of the complex stability function

\begin{tabular}{|c|c|}
\hline Function Value $\mathrm{y}(\mathrm{t})$ & Level of stability \\
\hline $0 \leq \mathrm{y}(\mathrm{t}) \leq 0,6$ & Critical state of organization \\
\hline $0,61 \leq \mathrm{y}(\mathrm{t}) \leq 0,73$ & Unstable state of the organization \\
\hline $0,74 \leq \mathrm{Y}(\mathrm{t}) \leq 1$ & Stable state of organization \\
\hline
\end{tabular}

The basis of calculations of the evaluation is to be based on the degree of compliance with real radar chart of an ideal chart, which represents the absolute stability in all factors. Geometrically, the value of the integral indicator for an ideal system can be compared to the area of the figure, which is a regular polygon (in the presented version - a pentagon) with a diagonal of 100 (Fig. 3).

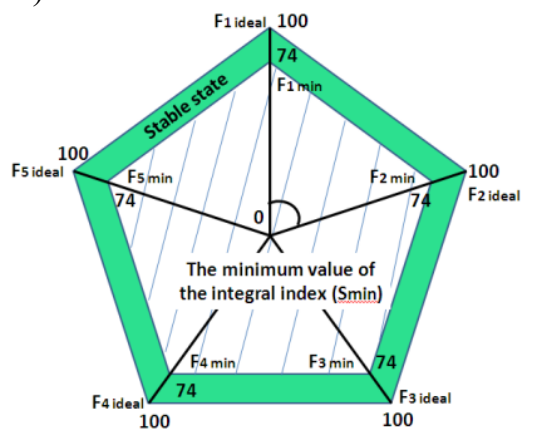

Fig. 3. Dynamics of factor values, reflecting the stability of the organization. 
The lower boundary of the stability zone will correspond to the area of the polygon with the diagonal $F_{i}$ min. The minimum value of the integral indicator of stability of the organization is calculated by the following formula:

$$
S_{\min }=\frac{1}{2} F_{i \min }^{2} * \sin \frac{360}{n} * n,
$$

where $F_{i \text { min }}$ is the minimum permissible value of the $\mathrm{i}$-th factor (not less than 0.74 ), $\mathrm{n}$ is the number of factors.

When determining the integral indicator of sustainability, the basic condition of the complex stability of the organization must be fulfilled - all factors entering the integral indicator should have a value of at least 0.74 . Only in this case it makes sense to evaluate the dynamics of the integral indicator. When assessing the sustainability of the organization, it is advisable to assess the state of complex sustainability in dynamics, which allows us to evaluate the correctness of management decisions, as well as monitor the dynamics of the organization's sustainable development:

$$
\Delta \mathrm{S}=\mathrm{S}_{\text {current }}-\mathrm{S}_{\text {previous }}
$$

where $S_{\text {current }}$ - the value of integrated sustainability in the current year,

$S_{\text {previous }}$ - the value of complex sustainability in the previous year.

If the value $\Delta S>0$, then the activity of the organization under consideration is effective and has a sustainable development.

Thus, assessing the results of the current period and comparing them with the results of the previous period, we can conclude on the level of sustainability of the activities of the organization, as well as the effectiveness of management decisions.

\section{Conclusions}

The approach proposed in the article can be seen as a useful tool for practical assessing and ensuring the sustainability of construction organizations and enterprises and as tool for theoretically predicting the future's needs along with the success of a business model.

\section{References}

1. E.V. Nezhnikova, The Economics of Construction Journal, 5(35), 14-21 (2015)

2. E. Nezhnikova, R. Obukhov, MATEC Web of Conferences, 106, 08025, (2017) https://doi.org/10.1051/matecconf /201710608025

3. D.N. Silka, M.A. Ivanova, Proceedings of Moscow State University of Civil Engineering, 12, 5(104), (2017)ndoi: 10.22227/1997-0935.2017.5.572-58

4. N.nOertwig, Life Cycle Engineering and Management (2017)

5. E. Nezhnikova, Proc.Eng, 165, 1300-1304 (2016) https://doi.org/10.1016/j. proeng.2016.11.854

6. H.M. Bernstein, M.A. Russo, D. Laquidara-Carr, Smart market report (2011) http://www.pepperlaw.com/resource/5452/0C0

7. N. Ivanov, MATEC Web of Conferences, 86, 05004 https://doi.org/10.1051/matecconf /20168605004

8. I.N. Aleksandrovich, Int. J. Appl. En. Res., 10, 42415-42418 (2015)

9. N. Ivanov, Proc. Eng., 153, 228-231 (2016) http://dx.doi.org/10.1016/j .proeng.2016.08.107

10. H. Allen Hu, S.H. Chen, C.W. Hsu, Int. J. Environ. Sci. Technol., 9-2, 343-354 (2012) https://doi.org/10.1007/s13762-012-0037-7 
11. K. Ishikawa, Productivity Press Taylor \& Francis (1990)

12. http://asq.org/learn-about-quality/cause-analysis-tools/overview/fish bone.html

13. V.N. Goncharov, E.A. Kolosova, Journal "Organizer of production, 3(58), 56-59 (2013)

14. I.G. Lukmanova, E.V. Nezhnikova, Proceedings of Moscow State University of Civil Engineering, 6, 158-164 (2014) https://doi.org/10.22227/1997-0935.2014.6.158-164 